Gesnerus 60 (2003) 220-234

\title{
Von Basel nach Bad Nassau. Das Schicksal des Psychoanalytikers Arthur Muthmann (1875-1957)
}

Thomas Müller

\section{Summary}

Arthur Muthmann is known as a pioneer of psychoanalysis in Germanspeaking countries. He published on the clinical application of psychoanalysis as well as an article explaining the matter to laymen. His Freiburg years and later work have already been portrayed, while his academic failure in Switzerland and his directorship of a private sanatorium at the famous Hessian spa of Bad Nassau have hardly been looked at. Historians of psychoanalysis reported on Muthmann's definite split from the psychoanalytic movement after 1909 due to the fiasco about his professoral thesis at Basel. His contribution to the spreading of psychoanalytic knowledge of the time has been judged upon harshly by known historians. Both hypotheses will be scrutinised here.

* Der Verfasser dankt Dr. Menachem Amitai, Freiburg i.Br., für die Übermittlung einer Reihe von Informationen zu Arthur Muthmann; Priv.-Doz. Dr. Michael Kutzer, Wiesbaden, für Literaturhinweise zu Bad Nassau in der Landesbibliothek Wiesbaden sowie Dr. Hans-Georg Hofer, Freiburg i.Br., für die kritische Diskussion im Vorfeld. Der Verfasser dankt darüber hinaus ausdrücklich Frau Dorothee Brown, Stadtarchiv Bad Nassau, für Hinweise zum Kurhaus Bad Nassau und Frau Angelika Dombrowsky, Bad Nassau, für Informationen zur Stadt Nassau. Bezüglich Hinweisen aus dem Stadtarchiv Freiburg i.Br. ist der Verfasser Frau StadtAOInsp. Hefele für die freundliche Unterstützung zu Dank verpflichtet.

Dr. Thomas Müller, Charité, Zentrum für Human- und Gesundheitswissenschaften (ZHGB), Institut für Geschichte der Medizin, Campus Benjamin Franklin, Klingsorstrasse 119, D-12203 Berlin (t.mueller@medizin.fu-berlin.de oder thomas.mueller@charite.de). 


\section{Zusammenfassung}

Arthur Muthmann gilt als Pionier der Psychoanalyse im deutschsprachigen Raum. Als früher Korrespondenzpartner Freuds veröffentlichte Muthmann eine Monographie zur Anwendung der Psychoanalyse sowie eine für Laien verständliche Schrift gleichen Inhalts. Muthmanns Beitrag zur Geschichte der Psychotherapie und Psychosomatik ist für seine Freiburger Zeit aufgearbeitet worden, nicht jedoch für seine Sanatoriumstätigkeit im hessischen Bad Nassau. Sein Engagement für die Psychoanalyse und der Versuch ihrer klinischen Integration an der Psychiatrischen Universitätsklinik in Basel zerstörten nachhaltig seine akademische Karriere. Muthmann migrierte zurück nach Deutschland und wurde Leiter einer Kurklinik, in der er selbst einmal Patient gewesen war. In der Historiographie wurde bisher von einer frühen und endgültigen Abwendung Muthmanns von der Psychoanalyse berichtet. Sein Beitrag zur Verbreitung der Psychoanalyse wurde aus dem Kreis der Freud-Biographen negativ beurteilt. Beide Hypothesen werden hier einer kritischen Revision unterzogen.

\section{Vorbemerkung}

Dieser Beitrag widmet sich zwei Aspekten: Zum einen war Arthur Muthmann eine beachtenswerte Figur in der Geschichte der Psychotherapie, worauf Menachem Amitai, der Muthmanns Freiburger Zeit bearbeitete, bereits hingewiesen hat ${ }^{1}$. Im ersten Teil des vorliegenden Beitrags wird im Anschluss an eine kurze biographische Einleitung Muthmanns Tätigkeit und Wirken in Bad Nassau an der Lahn aufgearbeitet und so die bisherige Lücke in der Biographierung dieses psychotherapeutischen Pioniers geschlossen. Im zweiten Teil, einem Beitrag zur Geschichte des privaten Krankenhauses, wird das Kurhaus in Bad Nassau in den Jahren unter der Leitung Arthur Muthmanns dargestellt ${ }^{2}$.

1 In bezug auf die Freiburger Jahre Muthmanns wird auf die ausführliche Arbeit Menachem Amitais (Amitai 1981) verwiesen, der noch zu Lebzeiten von Hans-Otto Muthmann Einsicht in die Lebenserinnerungen von dessen Vater Arthur Muthmann nehmen konnte (Muthmann, Arthur: Aufzeichnungen. Aus der Familiengeschichte, 1918/1919, unveröffentlicht). Hier werden lediglich einige Daten zum Verständnis aufgeführt sowie einige Informationen ergänzt, auf die Amitai noch nicht zurückgreifen konnte.

2 Der vorliegende Beitrag steht in Zusammenhang mit weiteren Arbeiten des Verfassers, deren gemeinsames Erkenntnisinteresse unter anderem in der Frage nach der Integration psychotherapeutischer Theorie und Praxis in die Schulmedizin der ersten Hälfte des 20. Jahrhunderts besteht. Siehe u.a. Müller 2000a; 2002, 102-119; Müller/Hermanns 2001 sowie zuletzt Müller 2004 (im Druck). 


\section{Zur Person}

Arthur Muthmann wurde am 24. April 1875 in Wuppertal-Elberfeld als zweitjüngster Sohn von zehn Kindern der Eltern Wilhelm und Emilie Muthmann, geb. Holzrichter, geboren ${ }^{3}$. Muthmanns Elternhaus war «streng protestantisch» ${ }^{4}$. Muthmanns Vater war Fabrikant. Arthur Muthmann studierte nach Erlangung der Hochschulreife 1893 bis 1894 zunächst Theologie in Tübingen und Erlangen. Dieses Studium wurde durch einen Kuraufenthalt im Sanatorium Dr. Poensgen in Bad Nassau an der Lahn unterbrochen - dem Ort seiner späteren Arbeitstätigkeit. Nach Beendigung der Kur und nach einem weiteren Semester Theologie in Bonn beschloss Muthmann gegen den Willen seiner Familie, Humanmedizin zu studieren ${ }^{5}$. Dieses Studium begann er 1896 in Bonn. Vier Wochen vor dem medizinischen Staatsexamen soll er eine «depressive Verstimmung» erlitten haben. Diese Erkrankung war offensichtlich ein Anlass für Muthmanns Interesse an der Psychiatrie und später der Psychoanalyse. 1899 zog Muthmann nach München um und approbierte 1900. Ab Ostern 1900 arbeitete Muthmann erneut in Bonn als Volontär bei Professor Köster im Pathologischen Institut der Universität. Im Herbst 1900 assistierte er drei Monate in einer Bonner «Irrenanstalt» ${ }^{6}$. Seine medizinische Promotion, während der er mit gesundheitlichen Beschwerden zu kämpfen hatte, schloss er mit summa cum laude dennoch sehr erfolgreich ab ${ }^{7}$. Nach der Verlobung 1907 hatte er die am 30. Mai 1878 in Berlin geborene Anna-Luise Charlotte Wilhelmine Freiin von der Goltz (gest. 1960) 1908 geheiratet. Arthur und Anna-Luise Muthmann hatten zwei Kinder ${ }^{8}$.

3 Die biographischen Daten zu Arthur Muthmann, zu den Wohnorten der Familie Muthmann sowie zu Carl Eugen Muthmann stammen aus dem Stadtarchiv Freiburg i.Br., Mitteilung an Verfasser vom 15. Februar und 1. März 2001, sowie aus dem Personenstandsbuch des Standesamts Wuppertal-Elberfeld, G. Haase, Mitteilung an Verfasser vom 13. März 2001.

4 Siehe Amitai 1981, 6-12. Zusammenfassung: Amitai/Cremerius 1984, 738-753.

5 Auf diesen Entscheidungsprozess ist bereits Amitai näher eingegangen: Amitai 1981, 9-10.

6 Die genaue Identität dieses Hauses konnte bisher nicht geklärt werden.

7 Die Dissertation trägt den Titel «Ein Fall von professioneller Parese im Peronealgebiet».

8 Wilhelma, die im Jahr der Heirat, 1908, geboren wurde, eine spätere Kinderärztin (1908 bis 1972), sowie den Sohn Hans-Otto (1910-1987), der zunächst Jurist wurde und - hier seinem Vater folgend - später auch archäologisch tätig war. Bei dem Priv.-Doz. Eugen Muthmann, der in Rostock und Göttingen als Anatom tätig war und dessen Name sich in den Ärzteverzeichnissen des Deutschen Reiches findet, handelt es sich offenbar um einen Bruder Arthur Muthmanns. Das Standesamt Wuppertal meldet die Geburt von Carl Eugen Muthmann, Sohn der nämlichen Eltern, am 12. März 1877. 


\section{Arthur Muthmann in Basel}

1901 arbeitete Muthmann zunächst in der Bonner Pathologie, bevor er 2. Assistent der Pathologie bei Prof. Kauffmann in Basel wurde. Von 1902 bis 1907 war er als Assistenzarzt an der Basler Psychiatrischen Universitätsklinik «Friedmatt» tätig, zunächst als Assistent von Prof. Wille. Bald darauf wurde er 2. Arzt von Prof. G. Wolff, der als ehemaliger Oberarzt Chefarzt geworden war. Mit Wolff kam es zu erheblichen Spannungen, weil Muthmann die psychiatrische Behandlung an der Friedmatt nach psychoanalytischen Prinzipien reformieren wollte 91905 hatte Muthmann Breuers und Freuds Studien über Hysterie studiert und versucht, die Erkenntnisse aus dieser Schrift in seiner klinischen Arbeit anzuwenden ${ }^{10}$. So gehörte Muthmann in den Kreis der frühen Freud-Rezeption im deutschsprachigen Raum, dessen Mitglieder häufig ärztlich-psychiatrisch tätig waren ${ }^{11}$. Er trat mit einer psychoanalytischen Buchpublikation (Psychologie und Therapie neurotischer Symptome) hervor, was ihm zu viel Sympathie unter den Psychoanalytikern gereichte. Der Freud-Adept Ernest Jones rezensierte das Buch sehr positiv und hatte darüber hinaus in diesem Brief an Muthmann vom 10. März 1908 seinem Wunsch Ausdruck verliehen, Muthmann auf dem 1. Internationalen Psychoanalytischen Kongress in Salzburg anzutreffen ${ }^{12}$. Muthmanns Habilitationsvorhaben bestand in nichts weniger als einem Forschungsbeitrag zur psychoanalytisch orientierten Therapie psychischer Erkrankungen. Ein ungewöhnlich früher Zeitpunkt für ein solches Unterfangen in Sachen Psychoanalyse, an dem in den nächsten beiden Dekaden jedoch noch weitere Ärzte scheiterten. Dem voraus ging die Publikation der genannten Monographie, in der er seinen Vorgesetzten Wolff ohne dessen Wissen namentlich mit der psychoanalytischen Arbeit in Verbindung brachte. Diese Publikation fand 1907 jedoch nicht nur die tiefe Abneigung von Wolff, der den Therapieversuchen mit einer Art «modifizierter Hypnoanalyse» (Hermanns) misstrauisch gegenüberstand, sondern sie führte über den Abbruch der Habilitation hinaus auch zur Beendigung von Muthmanns Tätigkeit in Basel ${ }^{13}$. Carl Gustav Jung und Sigmund Freud versuchten nachfolgend Muthmann davon zu überzeugen, nun seine gesamte Energie in die Weiterentwicklung

9 Siehe ausführlich bei Amitai 1981, 11-12.

10 Hermanns 1985, 20-21 und 181.

11 Hermanns 1994, 30-40.

12 Hermanns 1985, 20.

13 Amitai 1981, 12. Die Personal- und Verwaltungsakten der Zeit befinden sich nicht mehr im Archiv der Psychiatrischen Universitätsklinik. Prof. Dittmann, Mitteilung an Verfasser, 19. März 2001. Inwieweit sich im Staatsarchiv Basel noch relevante Akten befinden, konnte vor Drucklegung nicht mehr geklärt werden. 
der Psychoanalyse zu investieren. Muthmann liess jedoch, im Gegensatz zu einem offenbar anhaltenden Kontakt zu Jung ${ }^{14}$, seine Kontakte zur institutionalisierten Psychoanalyse nach einem Besuch bei Freud sowie der Teilnahme an einer Sitzung der Wiener Psychoanalytischen Vereinigung 1909 ruhen ${ }^{15}$. Eine Mitgliedschaft in dieser frühen Phase bestand nicht. Ernest Jones kommentierte in einem Nachruf auf Karl Abraham den «Rückzug» Muthmanns Jahre später in einem Ton, der deutlich weniger freundlich war als zuvor: «He [K. A.] was thus the first true psycho-analyst in Germany; for one can hardly call such the few men, Muthmann, Warda etc., who had gone only a little way with Freud's theories [...].» ${ }^{16}$ Bisher wurde berichtet, dass Muthmann 1909 - und zwar bleibend - den Kontakt zur institutionalisierten Psychoanalyse abgebrochen habe. Dies ist nicht zutreffend. Die hämische, jedenfalls kränkende Aussage Sigmund Freuds über Muthmann («Er reicht nicht an seinen Namen heran») soll Arthur Muthmann seinem Sohn zufolge allerdings nie erfahren haben ${ }^{17}$. Dies wirft neues Licht auf die Erklärungsversuche, wonach sich Muthmann auch deswegen von der institutionalisierten Psychoanalyse distanziert habe. Entgegen bisherigen Annahmen bedeutete das Jahr 1909 jedoch mitnichten den endgültigen Bruch Muthmanns mit der Psychoanalyse - auch nicht mit der institutionalisierten Psychoanalyse: Arthur Muthmann wurde 1937 ausserordentliches Mitglied der Deutschen Psychoanalytischen Gemeinschaft (DPG) ${ }^{18}$.

\section{Nach Bad Nassau an der Lahn}

Muthmann ging nach seiner Entlassung aus der Basler Psychiatrischen Universitätsklinik nach Deutschland zurück. Er wurde zunächst 2. Arzt in einem Sanatorium in der preussischen Provinz Hessen-Nassau, in dem er sich Jahre zuvor bereits einmal hatte behandeln lassen. In Bad Nassau an der Lahn, zwischen Westerwald und Taunus unweit Bad Ems ${ }^{19}$, war Muthmann ab 1907 in einem Kurhaus in privater Trägerschaft tätig, das aus einer 1856 von Emil

14 Freud/Jung 1974, 200-235 und 377.

15 Amitai 1981, 13-18.

16 Jones 1926, 155-181.

17 Hans-Otto Muthmann, der 1987 verstarb, soll Amitai zufolge auch ohne medizinische Ausbildung Expertise zur Beurteilung der Tätigkeit seines Vaters erworben haben. Hans-Otto Muthmann erfuhr von Freuds negativer Beurteilung des Beitrags seines Vaters aus der Freud-Biographie von Ernest Jones. Korrespondenz Muthmanns mit Menachem Amitai, 1974. Amitai, Freiburg, persönliche Mitteilung an Verfasser, 29. April und 8. Mai 2001.

18 Hier entnommen dem Korrespondenzblatt der Internationalen Psychoanalytischen Vereinigung 23 (1937) 586. Auch nach 1945 blieb er Mitglied der DPG.

19 Zur Balneologie in Bad Ems vergleiche Sommer/Hermann 1999. 
Haupt (1819-1866) gegründeten ${ }^{20}$ und von 1867 bis 1882 von Ferdinand Runge (gest. 1882) geleiteten «Kaltwasserheilanstalt» hervorging. Runge führte die Anstalt im Sinne einer Einrichtung für Wasserkuren und andere physikalische Heilmethoden weiter und publizierte wissenschaftlich zum Thema $^{21}$. Da unter Runges Leitung die Kaltwasserbehandlung zurücktrat, wurde das Kurhaus seit 1877 neutraler «Wasserheilanstalt» genannt. Nach kurzer Vertretung durch Geisse-Ems übernahm im April 1882 der Militärarzt Gustav Wolzendorff die ärztliche Leitung des Kurhauses, der aufgrund eines Nervenleidens nach drei Jahren den Dienst quittierte. Unter Wolzendorff war die Wasserbehandlung zugunsten der elektrischen Behandlung jedoch bereits in den Hintergrund getreten. In einer Publikation des Vorstands des Hauses von $1892^{22}$ werden folgende zur Behandlung kommende Erkrankungen genannt:

Rheumatische und gichtische Erkrankungen der Muskeln und Gelenke, Gelenkentzündungen, chronische Verdauungsstörungen (Magenleiden, Darmkatarrhe, Diarrhoe, habituelle Stuhlverstopfung), Circulationsstörungen [...] Nerven- und Rückenmarkskrankheiten (Neuralgien, Ischias, Lähmungen [...] Hysterie, Hypochondrie, Neurasthenie, Schwächezustände), Ernährungs-Anomalien [...] Die Neigung zu Erkältungen, zu Hals- und Lungenkatarrhen wird durch vorsichtige Abhärtungskuren zweckmässig bekämpft. Bei [...] Ueberarbeitung oder bei Erholungsbedürftigen ist neben den Kurmitteln namentlich die Ruhe und die geregelte diaetetische Lebensweise [...] von günstigem Einfluss. Geisteskranke werden nicht aufgenommen [...] Je nach Gesammtzustand des Patienten und nach der Natur des Leidens werden die vorhandenen Heilmittel einzeln benutzt oder [...] combinirt. Strengste Individualisirung ist dabei der hauptsächlichste Grundsatz [...]. ${ }^{23}$

Für die Zeit vor Muthmanns Eintritt ins Kurhaus Bad Nassau gilt also ohne Zweifel, dass die verschiedenen diätetischen Massnahmen und Wasserheilmethoden Vorrang vor anderen Therapieformen hatten ${ }^{24}$. Der im April 1883 in die Einrichtung eingetretene Eugen Poensgen, der mit einer Tochter

20 Die hierfür gegründete Aktiengesellschaft übertrug Haupt die Aufgabe, aus der von ihm «gegründeten und bisher betriebenen Heilanstalt für Kaltwasserbehandlung, Dampfbäder, Fichtennadeln, Electricität und Heilgymnastik eine Anstalt zu schaffen, die der Einrichtung [...] eines Pensionates für Touristen nach Art der Schweizer Pensionate» angeglichen werden sollte. Siehe Nassau (Bad) (o.J.).Zu Haupt: Schönrock 1998, 150-158. Von Haupt selbst liegen drei Publikationen aus 1857, 1858 und 1860 vor.

21 Auf die lesenswerten Beiträge kann hier nur verwiesen werden: Runge 1868; 1869a; 1869b, ${ }^{2} 1874 ; 1870 ; 1872,{ }^{3} 1877 ; 1879$ sowie 1881.

22 Die sich im Inhalt nicht nennenswert unterscheidet von:Verwaltungsrath des Kurhauses Bad Nassau 1887.

23 Vorstand des Kurhauses Bad Nassau 1892.

24 Hier finden Kiefernnadelbäder, römische Bäder (bei Rheumatismus und «nervösen Schmerzen»), Solbäder und «electrische Bäder» Anwendung - letztere namentlich bei Hysterie und Neurasthenie. An anderer Stelle in der gleichen Publikation erfährt der Leser, dass «schwere und hartnäckige Erkrankungen, namentlich Nervenleiden und Dyskrasien» längere Kuren von mindestens 7-8 Wochen erfordern würden. Es wird nicht versäumt zu erwähnen, dass einer der Vorzüge dieser Einrichtung in der «steten Anwesenheit» des Arztes bestehe. Es ist von «warmen und lauen Bädern, temperirten Abreibungen mit oder ohne vorherige Einpackungen, kühlen Halbbädern und Sitzbädern, Brausen und Douchen [...]» die Rede. 
Haupts verheiratet war, wurde im Dezember 1885 mit der Leitung beauftragt und führte eine umfassende Modernisierung der gesamten Einrichtung durch $^{25}$. Neben der Betonung der elektrischen Behandlung waren die Veränderungen Poensgens im wesentlichen ökonomisch-technischer Art. Im therapeutischen Sinn griff er auf bereits zuvor etablierte Heilmethoden zurück. Der Nutzen dieser Neuerungen ist für das Florieren und den ökonomischen Aufstieg des Kurhauses nicht zu unterschätzen, hier jedoch weniger relevant.

Die Situation, die Arthur Muthmann in Bad Nassau vorgefunden hat, lässt sich aus einer Publikation Poensgens an entlegener Stelle erschliessen ${ }^{26}$. Poensgen berichtete, dass schon zu Zeiten Haupts und Runges die Zahl der «nervösen Kranken» allmählich gewachsen war:

Mit der Ausbreitung der nervösen Krankheitszustände hat sich dann unser Haus immer mehr als vorwiegende Nervenheilanstalt entwickelt. Andere Krankheitsfälle wurden seltener und noch viel seltener fehlte ihnen die mehr oder weniger starke nervöse Färbung. Und je mehr die allgemeine nervöse Erregbarkeit anstieg, je mehr die früheren asthenischen Zustände sich in solche mit sehr gesteigerter Uebererregbarkeit wandelten, desto notwendiger und selbstverständlicher erwies sich die Aenderung in der Richtung der ärztlichen Massnahmen. ${ }^{27}$

Was Poensgen hier beschreibt, war für diese Zeit charakteristisch und schien diätetische Regeln ebenso wie Fragen der individuellen Haltung zu beinhalten: «Wir fanden von Jahr zu Jahr mehr unsere Hauptaufgabe in der psychischen Behandlung darin, dass der Kranke lernt, sich richtig zu leiten, möglichst viel Kräfte zu sammeln und zu bewahren, die Schädigungen [...] möglichst zu vermeiden, die Genüsse einzuschränken - und dadurch seinen Pflichten erhalten und gewachsen zu bleiben.» Psychotherapeutische Aspekte beinhalteten die von Poensgen erwähnten Heilmethoden jedoch nicht: «[...] Mit grosser Vorliebe [hingegen, Th. M.] findet die Elektrizität Anwendung in allen heute gebräuchlichen Formen [...].»

Bei Muthmanns Dienstantritt umfasste der immer wieder erweiterte Bau des Kurhauses «100 Logirzimmer und 6 grosse Gesellschaftsräume». Grosse

Die Kuren schlossen Behandlungen mit «Electricität» ein. Dies gilt auch für die Zeit von 1909 bis 1914 unter Muthmann, während der laut einer Broschüre des Hauses auch Behandlungen zur Anwendung kamen wie D'Arsonvalisation; Franklinisation; Elastomassage; Vibration; galvanische, faradische und Wechselstrombäder; Glühlicht- und Bogenlichtbad oder Diathermie.

25 Schönrock 1998, 155. Poensgen war in Strassburg bei Kussmaul in Innerer Medizin und in der Eberswalder Provinzial-Irrenanstalt bei August Zinn ausgebildet worden, Poensgen $1906,15$.

26 Dass Muthmann als seinerzeit psychoanalytisch orientierter Arzt den Dienst in einer ehemaligen Wasserheilanstalt antritt, stützt den bereits an anderer Stelle beschriebenen Befund, nach dem diese hohe Bedeutung für die Entwicklung der Privatsanatorien und damit der Psychoanalyse hatten. Siehe hierfür bei Shorter 1990, 159-195, sowie 1996, 320-333.

27 Vgl. hier und im folgenden Poensgen 1901 sowie 1906, 23-25. 
Veranden machten den Aufenthalt im Freien auch bei ungünstigem Wetter möglich, eine Zentralheizung gehörte bereits zur Grundausstattung. Die Leitung des Hauses hatte Muthmann Anfang Oktober 1908 übernommen ${ }^{28}$. Er war zunächst als 2. Arzt unter Eugen Poensgen tätig, neben dem dritten Arzt des Kurhauses, dem Stabsarzt a.D. Dr.A. Wagner. Poensgen trennte sich 1908 von seiner Ehefrau, die mit den Kindern in Bad Nassau blieb. Poensgen verliess Nassau, was der wesentliche Grund ist für die rasche Übernahme des Kurhauses durch Muthmann. Schönrock nennt Muthmann an entsprechender Stelle nicht namentlich, berichtet jedoch davon, dass die «Jahre bis zum Ersten Weltkrieg [...] für das Kurhaus Bad Nassau stetigen und regelmässigen Fortschritt» [brachten] und dass «Bad Nassau international an Anerkennung und Bedeutung» gewann ${ }^{29}$. In den Kriegsjahren von 1914 bis 1918 hatte Muthmann darüber hinaus die Funktion eines leitenden Arztes des Vereinslazaretts Nassau inne. Das Kurhaus der Geburtsstadt des Freiherrn vom und zum Stein, die damals nicht viel mehr als 2200 Einwohner hatte, ist ab 1910 im Jahrbuch der Heil-, Pflege- und Kuranstalten erwähnt. 1914 findet sich hier eine halbseitige Annonce mit Abbildung der Einrichtung für «diätetische Kuren». Es wird für die «neuzeitliche Einrichtung» des ganzjährig geöffneten Hauses geworben ${ }^{30}$, das drei Ärzte beschäftigte; leitender Arzt war Arthur Muthmann.

Prospekte sind bei der Verwaltung erhältlich [...]. Das Kurhaus empfiehlt sich als Aufenthalt für Ruhebedürftige und Rekonvaleszenten. Es kommen zur Behandlung innere Leiden und Nervenkrankheiten, Geisteskranke werden nicht aufgenommen. Die Behandlung ist eine individuelle, sie findet nach klinischen Grundsätzen statt. Behandlungsformen sind: Diätetische Kuren, Freiluftliegekuren bzw. Luftbäder, Massage, Hydrotherapie, Elektrizität (Apparate für Hochfrequenz- und Influenzbehandlung), alle Arten von Bädern, psychische Behandlung [usw.].

Bei diesem Kurhaus am Westrand Nassaus handelte es sich also um eine private allgemeine Heilanstalt für den gesellschaftlichen Mittelstand, wie sie Shorter beschrieb ${ }^{31}$ und für deren Innenleben eine eher luxuriöse, hotelähnliche Atmosphäre der Behaglichkeit ebenso charakteristisch war wie die «Medikalisierung der persönlichen Beziehungen» zwischen Patienten und

28 Im Nassauer Anzeiger vom 3. Oktober 1908 findet sich auf der Titelseite der Eintrag, dass «Mit dem heutigen Tage [...] Herr Sanitätsrat Dr. Poensgen und nach 25-jähriger segensreicher Tätigkeit als leitender Arzt des Kurhauses Bad Nassau von seinem Posten» zurücktrete. «Die Nachfolge als leitender Arzt ist dem bisherigen Assistenzarzt Herrn Dr. Muthmann übertragen.» Amtlich gemeldet in Bad Nassau findet man Muthmann 1906, ab 1909 mit einem Kind. Meldebücher Nassau, Dorothee Brown, Stadtarchiv Bad Nassau, persönliche Mitteilung an Verfasser, 22. Februar und 7. März 2001. Angelika Dombrowsky, Bad Nassau, Korrespondenz vom 13. Februar 2001.

29 Schönrock 1998, 155.

30 Neumann 1910, 33, sowie 1914, 47 (mit Fotografien) (Hervorhebung T.M.).

31 Hier und im folgenden Shorter 1996, 321 und 325. 
Ärzten oder die medizinische Disziplinierung des Tagesablaufs. Der Begriff «Krankenhaus» wurde tunlichst vermieden. Shorters Zahlenangaben zu den privaten Heilanstalten und Sanatorien sind zwar ergänzungsbedürftig, geben jedoch eine korrekte Tendenz der Entwicklung wieder: So trifft auch auf Nassau zu, was für die historische Wurzel vieler anderer privater Sanatorien gilt: Am Anfang stand eine Wasserheilanstalt.

Dieser Kureinrichtung, ihren Nachfolgemodellen und der zugehörigen Infrastruktur verdankte das an der späteren Schnellzugstrecke Berlin-KasselGiessen-Koblenz-Metz gelegene Städtchen seinen wirtschaftlichen Aufschwung vor allem im frühen 20. Jahrhundert. Während dieser Zeit war es (mit Ausnahme der Jahre des Ersten Weltkriegs) auch in Bad Nassau üblich, sogenannte Kurlisten in örtlichen Tageszeitungen zu veröffentlichen. Die zuletzt unter Eugen Poensgen veröffentlichte Kurliste ähnelt denjenigen unter Dr. Arthur Muthmann durchaus ${ }^{32}$ : Es finden sich Eintragungen zu Kurgästen aus den Niederlanden, aus England, Russland, Frankreich, Belgien, Luxemburg und den verschiedensten Gegenden des Deutschen Reiches ${ }^{33}$. Dies sowie die Berufsangaben und Namensbeisätze lassen die Interpretation zu, dass es sich meistenteils um Zugehörige gehobener bürgerlicher Schichten handelte. In einem Klinikprospekt hiess es, dass hypnotische und psychoanalytische Behandlung nur ausnahmsweise zur Anwendung käme. Aus der Zeit Muthmanns existiert eine weitere Quelle, der wiederum nur indirekt einige Informationen zur Krankenbehandlung um 1914 zu entnehmen sind ${ }^{34}$. Bei den «zur Behandlung im Kurhause» sich eignenden Erkrankungen werden an erster Stelle «die Nervenleiden» sowie «nervöse und organische Herzerkrankungen» genannt sowie eine Reihe weiterer Erkrankungen der Inneren Medizin.

Muthmann und seine Ehefrau zogen im Dezember 1919 nach Freiburg i.Br. Das Kurhaus Bad Nassau wurde von Dr. Rudolf Fleischmann (gest. 1945) und Dr. Fritz Poensgen (gest. 1936) übernommen, die es in den zwanziger Jahren betrieben ${ }^{35}$. Bei letzterem handelt es sich um den Sohn Eugen Poens-

32 Nassauer Anzeiger, 29. September und 1. Oktober 1908 sowie 21. Juli 1914 und 28. Oktober 1919.

33 In der Kurliste von 1914 finden sich darüber hinaus Gäste aus Chile, Italien und der Schweiz.

34 Kurhaus Bad Nassau (um 1914, mit umfassendem Bildmaterial), Stadtarchiv Nassau, Kopie beim Verfasser.

35 Der Nassauer Anzeiger vom 28. Oktober 1919 meldet, dass Fleischmann, bisher in Heidelberg, und Fritz Poensgen, bisher in Kiel, die ärztliche Leitung im Kurhaus übernehmen; ausserdem, dass der 2. Arzt, Sanitätsrat Wagner, eine leitende Arztstelle bei Erlenmeyer in der Anstalt Bendorf übernehme. Durch einen Bombenangriff 1945 wurde das Kurhaus fast völlig zerstört; nach dem Zweiten Weltkrieg wurde es nicht wieder aufgebaut. 
gens $^{36}$. Arthur Muthmann erscheint im Dezember 1920 in den Freiburger Einwohneradressbüchern ${ }^{37} .1934$ wird Muthmann in Freiburg als Arzt für Innere Krankheiten erwähnt ${ }^{38}$. Am 27. November 1944 wurde sein Sanatorium in Freiburg bombardiert und brannte $a b^{39}$. Muthmann starb am 8. Januar 1957, 82jährig, an einer akuten Pneumonie.

\section{Muthmanns Position im psychoanalytischen Feld}

In einschlägigen Publikationen wird Muthmann der frühen Freud-Rezeption zu Beginn des 20. Jahrhunderts zugerechnet. Entgegen der lange vorherrschenden Auffassung von der seitens der Schulmedizin marginalisierten Psychoanalyse $^{40}$ handelte es sich zunächst beim überwiegenden Teil dieser frühen Rezipienten um Ärzte ${ }^{41}$. Ohne eigene psychoanalytische Ausbildung erwarben diese Ärzte psychoanalytische Kenntnisse aus den frühen (vor-)psychoanalytischen Publikationen. Die zunächst probatorische Anwendung der Psychoanalyse wurde häufig ergänzt durch die Fachkorrespondenz bzw. die sequenzierte Besprechung von individuellen Therapien im Briefwechsel. Korrespondenzpartner waren in der Regel in der Anwendung der Psychoanalyse erfahrenere Praktiker bzw. Sigmund Freud selbst. Arthur Muthmann wurde schon früh als vielversprechender Propagator der Freudschen Therapie geschätzt. Der Zeitpunkt von Muthmanns Kontakt mit den

$36 \mathrm{Zu}$ den 1928 dort vordringlich behandelten Patienten, zu Personal und Ausstattung des Hauses siehe Verband Deutscher Ärztlicher Heilanstalts-Besitzer u. -Leiter 1928, 16, 74 (mit Fotografie des Kurhauses) und 114.

37 Amitai 1981, 19. Zu Muthmanns Patienten in Freiburg siehe Amitai 1981, 20, 28, 31-32. Er wird jedoch erst 1928/29 dort als Betreiber einer Klinik genannt, obwohl der Freiburger Bezirksrat bereits am 25. September 1919 eine Entschliessung an «den praktischen Arzt» Dr. Arthur Muthmann zur Erteilung der Genehmigung erliess, «nach Massgabe der vorgelegten Pläne und Beschreibungen, unter Aussch[1]uss [sic! Th. M.] von Geisteskranken und Patienten mit ansteckenden Krankheiten», im Haus Ludwigstrasse 36 ein Sanatorium zu betreiben. Stadtarchiv Freiburg im Breisgau, Aktennummer StadtAF C3/424/7.

38 In der Rubrik Nervenkrankheiten jedoch ist sein Name nicht vermerkt, während im Verzeichnis der Sanatorien der Stadt seine Einrichtung wiederum als Privathaus für «nervöse u. innere Kranke» erwähnt wird, mit beschränkter Krankenzahl (12 Betten), «am Schloßberg erhöht gelegen [...] Dr. A. Muthmann, früher leit. Arzt des Kurhauses Bad Nassau». Zum Freiburger Sanatorium: Amitai/Cremerius 1984, 749-752.

39 Bibliothek, wissenschaftliches Material und Kunstwerke wurden vernichtet. Bis April 1945 hielt sich das Ehepaar in Hinterzarten auf. Ab Juni 1945 wohnte Muthmann in der Holbeinstrasse 12, wo er noch einmal eine Praxis «für nervöse und innere Krankheiten» eröffnete.

40 Siehe Freud 1925, 31ff. Eine Arbeit, die diese Selbststilisierung am Beispiel Österreich untersuchte, ist: Tichy/Zwettler-Otte 1999, hier 33-38. Am Berliner Institut für Geschichte der Medizin wird eine Dissertation von D. Ricken mit dem Titel «Freud in der Tagespresse. Die Rezeption der Psychoanalyse in der bundesdeutschen Tagespresse zwischen 1950 und 1995» vom Verfasser mitbetreut.

41 Hier und im folgenden siehe wiederum in der Kurzbiographie von Hermanns 1985, 20. 
Studien über Hysterie wird mit spätestens 1905 angegeben. Bei seiner Publikation Psychiatrisch-theologische Grenzfragen. Historisches und Kritisches, die er (1907a) als 2. Arzt des Kurhauses Bad Nassau veröffentlichte ${ }^{42}$, handelte es sich um einen für das nichtmedizinische Publikum umgeschriebenen Beitrag, der als Fachpublikation mit dem Titel Zur Psychologie und Therapie neurotischer Symptome. Eine Studie auf Grund der Neurosenlehre Freuds im gleichen Jahr (1907b) erschienen war. In den erstgenannten Grenzfragen behandelte Muthmann auf vierzig Seiten die Entwicklung der «jungen Wissenschaft» der Psychiatrie ${ }^{43}$. Sein psychiatriehistorischer Überblick über das 19. Jahrhundert ist verlässlich und weist ihn jedenfalls als einen klinisch Tätigen aus, der die Vorgeschichte der Etablierung des Faches Psychiatrie, die zunächst optimistisch bewerteten Möglichkeiten der mikroskopischen Pathologie für die Psychiatrie wie auch andere zeitgenössische Fragestellungen in einen Kontext zu stellen versteht. Seine Bewertungen sind kritisch, seine Beobachtungen scharf, sein Stil hat Witz. Muthmanns Einschätzung der Möglichkeiten der psychiatrischen Therapie, auch unter Einbeziehung neuester Verfahren, kann aus heutiger Sicht als ausgewogen bis skeptisch bezeichnet werden. Freuds Beitrag zum Verständnis psychischer Erkrankung wird im Fazit sehr positiv bewertet. Muthmanns Verständnis der Entstehung sogenannter neurotischer Symptome ist umfassend. Das zeitgenössische psychoanalytische Verständnis der 〈Kunst〉, der 〈Religion> teilte er, psychoanalytische Fachtermini verstand er korrekt anzuwen$\mathrm{den}^{44}$. Der gerade Weg, sich dem Unbewussten zu nähern, ist für Muthmann 1905-1907 die Hypnose («Den Weg zum Unbewussten öffnet uns die Hypnose. In der Hypnose verkehren wir mit dem Unterbewusstsein des Menschen [...].»). Sein Gebrauch der Bezeichnung «psychoanalytisch» ist eher undogmatisch ${ }^{45}$.

Muthmanns zentrale Arbeit, die die grösste Aufmerksamkeit der zeitgenössischen Psychoanalytiker erhielt, war die Psychologie und Therapie neuro-

42 Darüber hinaus handelt es sich, wie der Autor in der Vorbemerkung erwähnt, um den «bis auf wenige Sätze», die hinzugefügt wurden, abgedruckten Beitrag in der Zeitschrift für Religionspsychologie 1 (1907).

43 Muthmann 1907a, hier 2, im folgenden 5-40.

44 Muthmann 1907a, 20-22. «Assoziation», «Retentionshysterie» oder «Abreagieren» und «Abfuhr» finden, wie viele andere mehr, Verwendung und werden an Beispielen (des Schriftstellers Ebers und des Philosophen Nietzsche) erklärt; auch solche psychoanalytischen Begriffe wie «Reminiszenz» werden erläutert, die bei Laplanche und Pontalis fehlen. Laplanche/Pontalis 1992.

45 «Zum Schlusse meiner Darlegungen möchte ich noch darauf hinweisen, dass die psychoanalytische Methode von geistlicher Seite unter religiöser Verbrämung schon gehandhabt worden ist $[\ldots] . »$ 
tischer Symptome (1907). Die klinischen Erfahrungen, auf denen diese Publikation beruht, stammten aus Muthmanns Zeit an der Basler Psychiatrischen Klinik 1905. Diese Ergebnisse kamen im Februar 1907 in Bad Nassau zur Niederschrift ${ }^{46}$. Anlass hierfür waren die «guten therapeutischen Erfahrungen», die Muthmann «mit der psychoanalytischen Methode machte». Er kannte die zeitgenössische psychiatrische Literatur zu Zwangserscheinungen, Assoziationsexperimenten oder «Sexualleben und Nervenleiden» und wusste sie zu den psychoanalytischen Schriften (v.a. Freuds bis 1906) in Beziehung zu setzen $^{47}$. Die Einleitung des Werks war mit Sicherheit geeignet, den interessierten Ärztinnen und Ärzten im Jahr 1907 eine gelungene, verständliche und anleitende Einführung in die zeitgenössische Psychoanalyse zu sein ${ }^{48}$.

\section{Schlussbemerkung}

Es wurde bereits darauf hingewiesen, dass in der Geschichtsschreibung der Psychoanalyse den im strengen Sinne psychoanalytischen Institutionen und Personen bisher vorrangig Aufmerksamkeit gewidmet wurde ${ }^{49}$. Untersucht man jedoch die Beziehungen zwischen Psychoanalyse und zeitgenössischer Schulmedizin, muss sich dieser Fokus weiten, zumal die alltägliche klinische Therapie und Praxis den wesentlicheren Teil des Fundaments zur Begründung von Freuds Theorie darzustellen scheint ${ }^{50}$. Bis in die Weimarer Jahre kommt hiermit den Beiträgen in Kliniken und Sanatorien Tätiger hohe Bedeutung zu. Wie an der Kureinrichtung des Kurhauses Bad Nassau zu sehen, dominierten zwar konventionelle balneologische, physikalische, elektrisch-mechanische Therapieverfahren, frühe psychotherapeutische Ansätze fielen jedoch auch hier auf fruchtbaren Boden. Arthur Muthmanns frühe Studie Zur Psychologie und Therapie neurotischer Symptome fusste auf klinischen Erfahrungen einer Zeit, in der er sich einer Art kathartisch orientierter Hypnoanalyse bediente (die er selbst psychoanalytische Me-

46 Muthmann 1907b, Vorwort I-III.

47 Über die hier genannten psychotherapeutischen Publikationen sind keine weiteren Publikationen zu diesem Thema bekannt. Hingegen publizierte Muthmann zusammen mit M. Hardtge 1933 das Werk Griechische Steinschriften als Zeichen lebendigen Geistes.

48 Dies kann ebenso für das Kapitel «Theoretisches» gesagt werden wie auch für das Kapitel zu Freuds «Sexualtheorie». Den überwiegenden Teil der Studie, insgesamt etwa sechzig Seiten, stellen das von Muthmann gesammelte Beobachtungsmaterial bzw. die Falldarstellungen dar. Im Vordergrund dieser Publikation stand der [1t. Muthmann «unschätzbare», Th. M.] Wert der Psychoanalyse, gerade in prophylaktischer Hinsicht.

49 Müller 2004 (im Druck).

50 Hier ist vor allem die Forschung von Ulrike May-Tolzmann zu nennen (1996). Siehe auch 1999, 769-781. 
thode nannte). Diese Therapieform unterschied sich einerseits grundsätzlich von der später entwickelten «klassischen» Psychoanalyse und musste noch ohne die Deutung von «Übertragung» und «Gegenübertragung» auskommen. Im Kern war die kathartische Methode eine Therapie der Hysterie. Dass jedoch «nichts an ihr erkennen liess, dass sich daraus ein theoretisches Wissen entwickeln könnte, das für alle Formen des Denkens, ob normal oder nicht, galt», wie es ein Freud-Biograph einmal formulierte ${ }^{51}$, trifft im Falle Arthur Muthmanns nicht zu. Muthmann war sich der Tragweite der «Entdeckungen» Sigmund Freuds, wie die Lektüre der Grenzfragen zeigt, durchaus bewusst - unabhängig davon, ob er später in praxi auf dem neuesten Stand psychoanalytischer Theorie blieb oder nicht -, was aufgrund fehlender Publikationen oder anderer Quellen auch kaum zu belegen sein dürfte ${ }^{52}$. Muthmann beendete, dem Vorwort zufolge, seine Arbeit an der Psychologie und Therapie im Februar 1907 in Bad Nassau. Zu dieser Zeit ist er bereits 2. Arzt des Kurhauses. Die Ursache von Muthmanns Fernbleiben vom psychoanalytischen Kongress in Salzburg im April 1908 ist zwar nicht bekannt, sein Fernbleiben bot jedoch Anlass zu negativen Spekulationen. Dass sein Schaffensschwerpunkt spätestens mit dem Oktober 1908 auf der ärztlichen Leitung des Nassauer Kurhauses liegen musste, bedarf keiner weiteren Erklärung. Mit einer Publikation für psychoanalytische Laien hat er sicherlich zu deren Verbreitung beigetragen. Für den Muthmann seitens Jones und Freud unterstellten Rückzug von der Psychoanalyse lassen sich keine Belege finden. Die bisher nicht bekannte Wiederannäherung an die Psychoanalyse in Form einer Mitgliedschaft zeigt im Gegenteil, dass die bisher ausschliesslich vertretene Beurteilung Muthmanns verdient, relativiert zu werden ${ }^{53}$. Die diffamierende Stellungnahme von Ernest Jones 1926, die getragen war von seiner Enttäuschung über einen Zeitgenossen, den er 1908 als Adepten und verlässlichen Fahnenträger der Psychoanalyse identifiziert zu haben glaubte, ist in jedem Falle problematisch. Jones' historiographische Bewertungen waren, was schon den Zeitgenossen auffiel ${ }^{54}$, häufig getragen von institutionspolitischen Motiven und machtpolitischem Kalkül und stellten jedenfalls keine Versuche dar, ein ausgewogenes Bild zu

51 Mannoni 1990, 36.

52 Neben den Studien über Hysterie konnte Muthmann bei der Niederschrift seiner Psychologie und Therapie theoretisch bereits auf weitere psychoanalytische Schriften zurückgreifen, u.a. Die Traumdeutung (1900), die Psychopathologie des Alltagslebens (1901), die Drei Abhandlungen zur Sexualtheorie, Der Witz und seine Beziehung zum Unbewussten, Bruchstück einer Hysterie-Analyse (alle drei 1905), vielleicht die Gradiva (1907).

53 Die Recherchen zu Muthmann sind Teil der Arbeiten an einem Biographischen Lexikon der Psychoanalyse in Deutschland 1908-1938. Für eine Projektskizze siehe Müller 2000b, 170-172.

54 Müller 2000a, 196-200. 
präsentieren. Da Jones’ Urteil im Rahmen eines Nachrufs publiziert wurde, dessen Zweck in der Heraushebung des Lebenswerks eines verlässlicheren Vertreters der psychoanalytischen Bewegung bestand (K. Abraham), wäre eine kritische Prüfung dieser Aussage um so notwendiger gewesen. Diese wenig kontextualisierte, retrospektiv und damit ahistorisch konstruierte Bewertung Jones' blieb jedoch lange die einzige Beurteilung des Beitrags von Arthur Muthmann.

\section{Literatur}

Amitai, Menachem, Arthur Muthmann. Ein Vorläufer der Psychoanalyse im Lande Baden (Freiburg i.Br. 1981)

Amitai, Menachem/Johannes Cremerius, «Arthur Muthmann. Ein Beitrag zur Frühgeschichte der Psychoanalyse», Psyche 28 (1984) 738-753

Freud, Sigmund, Selbstdarstellung. Gesammelte Werke Bd. XIV (Frankfurt a.M. 1925)

Freud, Sigmund/Carl Gustav Jung, Briefwechsel (Frankfurt a.M.1974)

Haupt, Emil, [Dr. Haupts] Heilanstalt zu Nassau a.d.L. (Wien 1857) sowie (Leipzig 1858)

- Der Schreibekrampf mit Rücksicht auf Pathologie und Therapie (Wiesbaden 1860)

Hermanns, Ludger M., «Frühe Freud-Rezeption am Beispiel Arthur Muthmann», in: Brecht, Karen/Volker Friedrich/Ludger M. Hermanns/Isidor J. Kaminer/D. H. Juelich (Hrsg.), «Hier geht das Leben auf eine sehr merkwürdige Weise weiter ...». Zur Geschichte der Psychoanalyse in Deutschland (Hamburg 1985) 20-21, 181

- «Karl Abraham und die Anfänge der Berliner Psychoanalytischen Vereinigung», LuziferAmor 13 (1994) 30-40

Jones, Ernest, «Karl Abraham 1877-1925», International Journal of Psycho-Analysis 7 (1926) 155-181

Kurhaus Bad Nassau (Hrsg.), Kurhaus Bad Nassau (ohne Verlagsangabe, wahrscheinlich 1914)

Laplanche, J./J.-B. Pontalis, Das Vokabular der Psychoanalyse (Frankfurt a.M. ${ }^{11} 1992$ )

Mannoni, Octave, Sigmund Freud (Reinbek 1971/1990)

May-Tolzmann, Ulrike, Freuds frühe klinische Theorie 1894-1896. Wiederentdeckung und Rekonstruktion (Tübingen 1996)

- "Freud's early clinical theory (1894-1896). Outline and context", International Journal of Psychoanalysis 80 (1999) 769-781

Müller, Thomas, Von Charlottenburg zum Central Park West. Henry Lowenfeld und die Psychoanalyse in Berlin, Prag und New York (Frankfurt a.M. 2000a)

- «Biographisches Lexikon der Psychoanalyse in Deutschland. Ein biographisches Nachschlagewerk von der Gründung der Berliner Psychoanalytischen Vereinigung 1908 bis zur Auflösung der Deutschen Psychoanalytischen Gesellschaft 1938. Unter Mitarbeit von Regine Lockot und Ludger M. Hermanns», Luzifer-Amor 26 (2000b) 170-172

- «Frieda Fromm-Reichmann. Jüdische Ärztin, ehemalige Dresdnerin, emigrierende Psychoanalytikerin, verfilmte Romanfigur», in: Scholz, Albrecht/Caris-Petra Heidel (Hrsg.), Medizin und Judentum, Bd. 6 (Frankfurt a.M. 2002) 102-119

- «Die Neurologische Abteilung des Krankenhauses Lankwitz. Ein Beitrag zur Geschichte des Wissenschaftstransfers, der Emigration und des Berliner Krankenhauses», Sudhoffs Archiv 1 (2004; im Druck)

Müller, Thomas/Ludger M. Hermanns, «Margarete Stegmann - Psychoanalytikerin, Reichstagsabgeordnete u. Frauenrechtlerin», Luzifer-Amor 27 (2001) 36-59

Muthmann, Arthur, Psychiatrisch-theologische Grenzfragen. Historisches und Kritisches (Halle a.d.S. 1907a)

- Zur Psychologie und Therapie neurotischer Symptome. Eine Studie auf Grund der Neurosenlehre Freuds (Halle a.d.S. 1907b) 
Nassau Bad (Hrsg.), Statuten der Actiengesellschaft des Bades Nassau (Nassau a.d.L., o.J., wohl jedoch vor 1869)

Neumann, H. (redig. von), Jahrbuch der Heil-, Pflege- und Kuranstalten (Privat-Anstalten) (Berlin 1910, 1914)

Poensgen, Eugen, «Beitrag zur Behandlung nervöser Krankheitszustände in Sanatorien», Zeitschrift für diätetische und physikalische Therapie 4 (1901)

- Kurhaus Bad Nassau 1856-1906. Fest-Schrift zum 1. Juni 1906 (München 1906)

Runge, F[erdinand], Der Rheumatismus der Muskeln und Gelenke (Wiesbaden 1868)

- Beobachtungen a. d. Gebiete der Kaltwasser-Kuren u.d. sog. Naturheilmethoden (Wiesbaden 1869)

- Kurmethoden u. Kurerfolge i. d. Badeanstalt zu Nassau f. Hydro- u. Elektrotherapie (Frankfurt 1870)

- Das Verhalten bei Bade- u. Kaltwasserkuren (Wiesbaden ${ }^{2} 1874$ )

- Beobachtungen a. d. Gebiete d. Wasserkuren u. anderer physikal. Heilmethoden (Wiesbaden ${ }^{3} 1877$ )

- Die Wasserkur. Allgemein verständliche Darstellung des Wesens und der Aufgaben (Leipzig 1879)

- Kurze Anleitung zum Gebrauche der Wasserkuren (Berlin 1881)

Schönrock, Karl-Heinz, «Dr. Emil Haupt und das «Kurhaus Bad Nassau»», in: Stadt Nassau (Hrsg.), Ursprung und Gestaltung. Geschichte und Geschichten (Nassau 1998) 150-158

Shorter, Edward, "Private Clinics in Central Europe 1850-1933", Social History of Medicine 3 (1990) 159-195

- «Heilanstalten und Sanatorien in privater Trägerschaft, 1877 bis 1933», in: Labisch, Alfons/Reinhard Spree (Hrsg.), Einem jeden Kranken in einem Hospitale sein eigenes Bett. Zur Sozialgeschichte des Allgemeinen Krankenhauses in Deutschland im 19. Jahrhundert (Frankfurt a.M./New York 1996) 320-333

Sommer, Hermann, Zur Kur nach Ems. Ein Beitrag z. Geschichte der Badereise von 1830-1914 (Stuttgart 1999)

Tichy, Marina/Sylvia Zwettler-Otte, Freud in der Presse. Rezeption Sigmund Freuds und der Psychoanalyse in Österreich 1895-1938 (Wien 1999)

Verband Deutscher Ärztlicher Heilanstalts-Besitzer und -Leiter (Hrsg.), Jahrbuch der ärztlich geleiteten Heilanstalten und Privatkliniken Deutschlands 1928 (Berlin 1928 sowie 1932)

Verwaltungsrat[h] d. Kurhauses Bad Nassau (Hrsg.), Wasserheilanstalt. Dirig. Arzt:Dr. Poensgen (Nassau 1887)

Vorstand des Kurhauses Bad Nassau (Hrsg.), Wasserheilanstalt. Dirig. Arzt: Dr. E. Poensgen (Hamburg 1892) 